\title{
Impact of Insulin Initiation and Time to Insulin Initiation on within-Person Change of Healthcare Utilization in Medicaid Enrollees with type 2 Diabetes
}

\author{
Lingshu Xue \\ Elsa S. Strotmeyer \\ University of Pittsburgh \\ Janice Zgibor \\ University of South Florida \\ Tina Costacou \\ University of Pittsburgh \\ Robert Boudreau \\ University of Pittsburgh \\ David Kelley \\ Pennsylvania Department of Human services \\ Julie M. Donohue ( $\square$ jdonohue@pitt.edu ) \\ https://orcid.org/0000-0003-2418-6017
}

University of Pittsburgh https://orcid.org/0000-0001-8226-6272

Research article

Keywords: type 2 diabetes, healthcare utilization, time to insulin use

Posted Date: July 14th, 2020

DOI: https://doi.org/10.21203/rs.3.rs-41114/v1

License: (a) (i) This work is licensed under a Creative Commons Attribution 4.0 International License.

Read Full License 


\section{Abstract}

Background: Insulin use, time to insulin initiation, and subsequent healthcare utilization in low-income adults with type 2 diabetes (T2DM) are poorly understood.

Methods: Our objectives were to examine whether 1) insulin initiation and 2) every 1-year decrease in the time from first-line glucose-lowering agents (GLAs) to insulin initiation reduced healthcare utilization within 12 months after insulin initiation in Pennsylvania (PA) Medicaid enrollees with T2DM. We included a cohort of 12,648 PA Medicaid enrollees (age 47.3 \pm 10.3 years) with first-line non-insulin GLAs between 2008 and 2016. Insulin users $(N=3,625)$ were matched with non-insulin users $(N=9,023)$ on dates of $1^{\text {st }}$ GLA prescription and propensity scores calculated based on baseline characteristics to account for potential confounders. Generalized estimating equations models estimated within-person changes in inpatient stays and emergency room (ER) visits 12 months after vs. 12 months before insulin initiation. We performed sensitivity analyses in young (18-45 years) and middle-aged enrollees ( $>45$ to 64 years).

Results: The average time from first-line GLAs to insulin initiation was $2.0 \pm 1.7$ years. Neither insulin initiation (rate ratio [RR]:1.0 [1.0,1.1]) nor time to insulin initiation (RR: $1.0[1.0,1.0]$ ) was associated with within-person change in ER visits. In young T2DM adults, insulin users had a greater subsequent increase in inpatient stays after insulin initiation vs. non-insulin users over the same time period (RR: 1.3 [1.1, 1.5]).

Conclusions: In T2DM Medicaid enrollees, no reduction in healthcare utilization was observed after insulin initiation, even in early stages of pharmacotherapy. Studies investigating primary contributors to the increased inpatient use in young insulin users with T2DM are needed.

\section{Background}

Medicaid, as the primary health insurance program for low-income people in the US, covered about $8 \%$ of all individuals with diagnosed diabetes in 2009 (approximately 1.8 million adults) [1, 2]. In 2012, nearly $13 \%$ of total healthcare expenditures for diabetes was provided by Medicaid ( $\$ 28$ billion) [3]. For each Medicaid enrollee with T2DM, the age-adjusted annual medical cost was $\$ 14,170$ in 2012, higher than $\$ 9,560$ in those with commercial insurance [1], with the annual medical cost increasing to $\$ 17,830$ for those with a diagnosis code of uncontrolled diabetes $(\mathrm{HbA} 1 \mathrm{c}>9 \%)$ possibly due to a higher prevalence of diabetes complications [1]. Of Medicaid enrollees with T2DM, only 64\% received annual $\mathrm{HbA} 1 \mathrm{c}$ test and even a lower portion (58\%) had controlled diabetes in 2012 [1]. Improving glycemic control with insulin use may therefore be important in reducing medical costs and healthcare utilization $[4,5]$.

Insulin initiation in the early progression of diabetes shows benefit for maintaining pancreatic beta-cell function [6] and reducing the risk of long-term microvascular complications by $24 \%$ in individuals with T2DM [7]. In 2012, 29\% of Medicaid enrollees with diagnosed T2DM used insulin and the total amount of insulin reimbursement substantially rose by $462 \%$ from 2006 to 2014 [1, 8]. Some studies conducted in managed care organizations showed a subsequent reduction in inpatient service after insulin initiation [9, 
10]. However, few studies have compared this reduction with those using non-insulin GLAs and addressed the effect of time to insulin initiation on the subsequent change of healthcare utilization in Medicaid enrollees, who have low-income, and a higher prevalence of disability and behavioral health and medical comorbidities. Our objectives were to examine whether (1) insulin initiation decreased the number of inpatient stays and ER visits within 12 months after insulin initiation; and (2) if a shorter length of time to insulin initiation from first-line GLAs was associated with reduction in inpatient stays and ER visits within 12 months after vs. before insulin initiation in young and middle-aged Medicaid enrollees with T2DM.

\section{Methods}

\section{Data source and study population}

We obtained a Medicaid administrative claims database from the Pennsylvania Department of Human Services (PADHS) to identify the study population. The database includes demographic characteristics, pharmacy claims, and ICD9/10-CM diagnosis codes, encounters with procedure information from inpatient and outpatient settings for all Medicaid enrollees in Pennsylvania (PA) from 2007 to 2016. The study sample for the cohort was limited to 168,594 Medicaid enrollees who had at least one prescription fill for non-insulin GLAs (i.e. sulfonylurea, thiazolidinedione, inhibitors of dipeptidyl peptidase 4, sodiumglucose co-transporter-2 inhibitor, glucagon-like peptide 1 receptor agonists) from January 1, 2008 through December 31, 2016 [2] (Fig. 1). From that group, we selected 136,998 Medicaid enrollees aged 18-64 years old who were not dually eligible for Medicare on the index date (i.e. the date of the first prescription fill for non-insulin GLAs) since pharmacy claims from Medicare were not available. To identify an incident cohort of new GLA users and allow for adequate measurement of healthcare utilization before insulin initiation, we further limited the study cohort to 45,560 enrollees with $\geq 365$ days of continuous Medicaid enrollment preceding the index date and without any prescription fills for GLAs in 2007. To exclude those with type 1 or gestational diabetes, we removed enrollees if a) they had no claims with T2DM diagnostic codes (ICD-9 250 or ICD-10 E11) in any position within 6 months before or after the index date $(\mathrm{N}=12,686)$; or $\mathrm{b})$ were women with a birth or a terminated pregnancy within 6 months before or after the index date $(\mathrm{N}=536)$. To allow for adequate measurement of healthcare utilization in the follow-up, we excluded enrollees without $\geq 890$ days ( 2.5 years) of continuous enrollment after the index date (the date of first-line GLAs). Additionally, insulin users without $\geq 365$ days of continuous enrollment after the date of insulin initiation were excluded. A cohort of 15,356 Medicaid enrollees with T2DM was used for matching analyses (Fig. 1).

\section{Matching}

The cohort of 15,356 Medicaid enrollees were followed from the index date through the first occurrence of any censoring event. Censoring events included the end of enrollment in Pennsylvania Medicaid, gaining dual eligibility for Medicare, or all-cause mortality. Insulin users $(N=3,715)$ were defined as enrollees with 
$\geq 1$ prescription fills for insulin during the follow-up period. The date of insulin initiation was the date of the first prescription fill for any type of insulin after the index date. Those without insulin prescriptions in the follow-up period, defined as non-insulin users, served as the comparison group $(N=11,641)$.

We used a hierarchical 1:3 matching strategy to assign a time point for non-insulin users to measure within-person change in healthcare utilization as well as to account for potential confounders of the association between insulin initiation and healthcare utilization (Fig. 2). Non-insulin users were matched with insulin users on a) the index date (the date of first line GLAs) \pm 5 days, b) continuous enrollment within 12 months before and after the date of insulin initiation, and c) propensity score (PS) with a deviation of 0.05 on the PS scale. The PS represented the probability of initiating insulin, which was estimated using a multivariable logistic regression model adjusted for covariates observed within 12 months before or on the date of first line GLAs. Covariates in the propensity score model included age, sex, race (White, Black, other), calendar year of the index date, primary enrollment in fee-for-service (as opposed to managed care), Medicaid eligibility categories (disabled or chronically ill vs. others such as Temporary Assistance for Needy Families) [11]. Our cohort effectively excluded the expansion group under the Affordable Care Act (ACA) which was implemented in 2015 in PA due to the continuous enrollment criteria we imposed. We also included the Area Deprivation Index, an area-based measure of education, income, and occupation status, using the 9-digit ZIP code of residence[11]. Additional covariates in the propensity score model measured within 12 months before first-line GLAs included indicators for the use of certain medications (anti-hypertensive agents, anticoagulant agents, lipid lowering agents, nitrates, and loop diuretics), indicators for the presence of diabetes-related comorbidities and complications (e.g. hypertension, obesity, depression, psychosis, congestive heart failure, nephropathy, neuropathy, retinopathy, cardio/cerebrovascular complications, and metabolic complications) [12], as well as a modified Elixhauser comorbidity index excluding the health conditions mentioned above that were included as separate indicators[13] (definitions in Additional file 1).

A total of 3,625 insulin users were matched with 9,023 non-insulin users based on the date of first-line GLAs, continuous enrollment and propensity scores. A total of 2,708 enrollees (90 insulin users and 2,618 non-insulin users) were excluded after matching since the distance of the PS within matched sets was either greater than 0.05 or greater than the first three smallest distances of the PS among all possible pairings [14]. After matching, no significant differences were noted in sex, psychoses, congestive heart failure and cardiovascular complications between insulin users and their matched non-insulin users indicating that the matching process attenuated these differences between groups, though other differences in race, Fee-For-Service status, prevalence of microvascular complications and depression, use of lipid-lowering agents, and healthcare utilization remained significant (Additional file 2).

\section{Primary Independent Variables}

The primary exposures were the binary indicator of insulin initiation (insulin users/non-insulin users) and the continuous variable for the time from first-line GLAs to insulin initiation, calculated by subtracting the 
index date from the date of the first prescription fill for insulin. For non-insulin users, the time to insulin initiation was calculated by subtracting the index date from the date of insulin initiation for respectively matched insulin users.

\section{Outcomes}

The primary outcomes were within-person change in the number of all-cause inpatient stays and ER visits after insulin initiation or the matched date. For insulin users, the numbers of inpatient stays and ER visits were assessed within 12 months before and 12 months after the date of the first prescription fill for insulin. For non-insulin users, the outcome was assessed 12 months before and 12 months after the date of insulin initiation for the matched insulin user. The number of inpatient stays was counted based on non-adjacent inpatient claims [15]. Adjacent inpatient claims with the same discharge and admission date were considered as hospital transfers, which were defined as a single hospital stay for the purpose of generating the count [15]. ER visits were determined by the outpatient claims with revenue center codes of $0450-0452,0456,0459,0981$ or procedure codes of 99281-99285, G0380, G0381-G0385[16]. ER visits which occurred in the period between the admission and discharge dates of any inpatient stays were excluded.

\section{Statistical analysis}

Descriptive statistics were conducted for enrollees' demographics, comorbidities, complications, and medication use measured on the date of first-line GLAs or within 12 months before first-line GLAs. Univariate comparisons between matched insulin users and non-insulin users were conducted using McNemar's tests for categorical variables, paired t-test for continuous variables with normal distribution, and Wilcoxon Rank sum tests for non-normally distributed continuous variables. The $\chi^{2}$ tests and $t$ tests were used to compare categorical and continuous variables in unmatched cohorts. Given that the number of inpatient stays and ER visits before and after insulin initiation were discrete and over-dispersed, generalized estimating equations (GEE) models with a negative-binomial distribution were used. The effect of time to insulin initiation after first-line GLAs on within-person change was examined in insulin users only. In order to attenuate the confounding effect between measured covariates and the withinperson change, interaction terms between time and covariates included in the propensity score were added to the model stepwise and removed at $p>0.1$ except for age, sex, and race. Collinearity was tested before adding covariates into the model. We also performed stratified GEE models for subgroups of enrollees aged 18 to $\leq 45$ years old (young adults) and from $>45$ to 64 years old (middle-aged adults) since we observed the interaction term between time and age significantly attenuated the relative risk ratio of within-person change in inpatient stays toward the null in the primary analysis. A sensitivity analysis was performed in the subgroup of those with 2 or more prescription fills for insulin vs. noninsulin users to exclude those who initiated insulin by chance. Analyses were performed using SAS, version 9.4 (SAS Institute, Inc., Cary, NC). 


\section{Results}

Baseline characteristics of insulin vs. non-insulin users in the matched cohort $(N=12,648)$ are shown in Table 1. After matching, insulin users $(N=3,625)$ and non-insulin users $(N=9,023)$ were different in several baseline characteristics. In the matched cohort, insulin users were 2 years younger (mean age: $45.6 \pm 10.1$ vs. $47.6 \pm 10.6$ years, $P<.001)$ and more likely to be Black $(32.3 \%$ vs. $28.9 \%, P<.001)$ than non-insulin users. Insulin users were more likely to have depression, psychosis, congestive heart failure, nephropathy and metabolic complications than non-insulin users. Insulin users were less likely to use lipid lowering agents compared to the matched non-insulin users. Insulin users had a higher number of inpatient stays $(0.5$ vs. $0.3 ; P<.001)$ and ER visits per person ( 1.9 vs. $1.4 ; P<.001)$ during the year before first line GLAs than matched non-insulin users. Stratifying by age, baseline characteristics were similar between insulin and non-insulin users in young adults $(N=4,745)$; whereas in middle-aged adults $(N=$ 7,903), insulin users were more likely to have depression, congestive heart failure, metabolic complications, and cardiovascular complications than non-insulin users (Additional file 3). 
Table 1

Patients characteristics on the index date or in 1-year before the index date

\section{Characteristics}

1:3 Matched cohort

Insulin users

$N=3625 \S$
Non-insulin users

$N=9023$

Demographic characteristics

Age, Mean(SD)

$45.6(10.1)^{\dagger}$

$47.6(10.6)$

Female, $\mathrm{N}(\%)$

$2431(67.1 \%)$

$5917(65.6 \%)$

Race, N (\%)

Non-Hispanic white

$1830(50.5 \%)^{\dagger}$

$4785(53.0 \%)$

Non-Hispanic black

$1171(32.3 \%)^{\dagger}$

$2605(28.9 \%)$

Hispanic

$513(14.2 \%)$

$1248(13.8 \%)$

Others

$111(3.1 \%)^{\dagger}$

$385(4.3 \%)$

FFS, N (\%)

$1083(29.9 \%)^{\dagger}$

$2462(27.3 \%)$

Disabled, N (\%)

$2835(78.2 \%)$

$112.4(7.7)$

$7196(79.8 \%)$

Area deprivation index, Mean(SD)

$5.7(1.8)^{\dagger}$

$112.1(8.0)$

Length of follow-up, Mean(SD)

$5.7(4.1,7.3)^{\dagger}$

$5.2(1.8)$

Length of follow-up, Median(IQR)

Index year, N (\%)

2008

$735(20.3 \%)^{\dagger}$

$1448(16.0 \%)$

IQR: interquartile range

FFS: fee-for-service

ER: emergence room

${ }^{\dagger} \mathrm{P}$-value $<.01$

* P-value $<.05$

₹ Modified Elixhauser Comorbidity Index evaluated 22 comorbidities, excluding hypertension, obesity, depression, psychoses, congestive heart failure, diabetes and diabetes related complications which are shown separately

$\S$ McNemar's tests for categorical variables, paired t-test for continuous variables with normal distribution, and Wilcoxon Rank sum tests for non-normally distributed continuous variables 


\begin{tabular}{|lll|}
\hline Characteristics & \multicolumn{2}{l}{$1: 3$ Matched cohort } \\
\cline { 2 - 3 } & Insulin users & Non-insulin users \\
& $\mathbf{N}=3625 \S$ & $\mathbf{N}=9023$ \\
\hline 2009 & $658(18.2 \%)^{\dagger}$ & $1469(16.3 \%)$ \\
\hline 2010 & $581(16.0 \%)$ & $1518(16.8 \%)$ \\
\hline 2011 & $564(15.6 \%)$ & $1512(16.8 \%)$ \\
\hline 2012 & $524(14.5 \%)$ & $1436(15.9 \%)$ \\
\hline 2013 & $402(11.1 \%)^{\dagger}$ & $1171(13.0 \%)$ \\
\hline 2014 & $161(4.4 \%)$ & $469(5.2 \%)$ \\
\hline Health conditions & & $1.4(1.5)$ \\
\hline Modified Elixhauser index, Mean(SD)‡ & $1.6(1.6)^{\dagger}$ & $5715(63.3 \%)$ \\
\hline Hypertension, N (\%) & $2204(60.8 \%)^{\dagger}$ & $2994(33.2 \%)$ \\
\hline Obesity, N (\%) & $1242(34.3 \%)$ & $2623(29.1 \%)$ \\
\hline Depression, N (\%) & $1124(31.0 \%)^{*}$ & $3381(37.5 \%)$ \\
\hline Psychoses, N (\%) & $1417(39.1 \%)$ & $516(5.7 \%)$ \\
\hline Congestive heart failure, N (\%) & $242(6.7 \%)^{*}$ & $390(4.3 \%)$ \\
\hline Diabetes complications, N (\%) & $188(5.2 \%)^{*}$ & $(11.6 \%)$ \\
\hline Nephropathy & $456(12.6 \%)$ & \\
\hline Neuropathy & & 1050 \\
\hline
\end{tabular}

IQR: interquartile range

FFS: fee-for-service

ER: emergence room

${ }^{\dagger} \mathrm{P}$-value $<.01$

* P-value $<.05$

¥ Modified Elixhauser Comorbidity Index evaluated 22 comorbidities, excluding hypertension, obesity, depression, psychoses, congestive heart failure, diabetes and diabetes related complications which are shown separately

$\S$ McNemar's tests for categorical variables, paired t-test for continuous variables with normal distribution, and Wilcoxon Rank sum tests for non-normally distributed continuous variables 


\begin{tabular}{|c|c|c|}
\hline \multirow[t]{3}{*}{ Characteristics } & \multicolumn{2}{|c|}{ 1:3 Matched cohort } \\
\hline & Insulin users & Non-insulin users \\
\hline & $N=3625 \S$ & $N=9023$ \\
\hline Retinopathy & $110(3.0 \%)$ & $264(2.9 \%)$ \\
\hline Peripheral Vascular Disease & $235(6.5 \%)^{\star}$ & $492(5.5 \%)$ \\
\hline Metabolic complications & $44(1.2 \%)^{\dagger}$ & $36(0.4 \%)$ \\
\hline Cardiovascular complications & $672(18.5 \%)$ & $1566(17.4 \%)$ \\
\hline Cerebrovascular complications & $173(4.8 \%)$ & $405(4.5 \%)$ \\
\hline \multicolumn{3}{|l|}{ Use of medication, $\mathrm{N}(\%)$} \\
\hline Anti-hypertensive medications & $1527(42.1 \%)^{\star}$ & $4025(44.6 \%)$ \\
\hline Anticoagulants or platelet inhibitors & $279(7.7 \%)$ & $699(7.7 \%)$ \\
\hline Statin and other lipid lowering agents & $1344(37.1 \%)^{\dagger}$ & $3974(44.0 \%)$ \\
\hline Nitrates & $145(4.0 \%)$ & $367(4.1 \%)$ \\
\hline Loop Diuretics & $438(12.1 \%)$ & $999(11.1 \%)$ \\
\hline \multicolumn{3}{|l|}{ Healthcare utilization } \\
\hline All-cause hospitalization, N (\%) & $1049(28.9 \%)^{\dagger}$ & $1946(21.6 \%)$ \\
\hline Number of inpatient stays, Mean (SD) & $0.5(1.0)^{\dagger}$ & $0.3(0.8)$ \\
\hline Number of inpatient stays, Median (IQR) & $0.0(0.0,1.0)^{\dagger}$ & $0.0(0.0,0.0)$ \\
\hline All-cause ER visits, N (\%) & $2097(57.8 \%)^{\dagger}$ & $4545(50.4 \%)$ \\
\hline
\end{tabular}

IQR: interquartile range

FFS: fee-for-service

ER: emergence room

${ }^{\dagger} \mathrm{P}$-value $<.01$

* P-value $<.05$

¥ Modified Elixhauser Comorbidity Index evaluated 22 comorbidities, excluding hypertension, obesity, depression, psychoses, congestive heart failure, diabetes and diabetes related complications which are shown separately

$\S$ McNemar's tests for categorical variables, paired t-test for continuous variables with normal distribution, and Wilcoxon Rank sum tests for non-normally distributed continuous variables 


\begin{tabular}{|c|c|c|}
\hline \multirow[t]{3}{*}{ Characteristics } & \multicolumn{2}{|c|}{ 1:3 Matched cohort } \\
\hline & Insulin users & Non-insulin users \\
\hline & $N=3625 \S$ & $N=9023$ \\
\hline Number of ER visits, Mean (SD) & $1.9(3.3)^{\dagger}$ & $1.4(2.8)$ \\
\hline Number of ER visits, Median (IQR) & $1.0(0.0,2.0)^{\dagger}$ & $1.0(0.0,2.0)$ \\
\hline All-cause outpatient visits, N (\%) & $3392(93.6 \%)^{\dagger}$ & $8718(96.6 \%)$ \\
\hline \multicolumn{3}{|l|}{ IQR: interquartile range } \\
\hline \multicolumn{3}{|l|}{ FFS: fee-for-service } \\
\hline \multicolumn{3}{|l|}{ ER: emergence room } \\
\hline \multicolumn{3}{|l|}{${ }^{\dagger}$ P-value $<.01$} \\
\hline \multicolumn{3}{|l|}{ * P-value $<.05$} \\
\hline \multicolumn{3}{|c|}{$\begin{array}{l}\text { ‡ Modified Elixhauser Comorbidity Index evaluated } 22 \text { comorbidities, excluding hypertension, obesity } \\
\text { depression, psychoses, congestive heart failure, diabetes and diabetes related complications which } \\
\text { are shown separately }\end{array}$} \\
\hline \multicolumn{3}{|c|}{$\begin{array}{l}\text { § McNemar's tests for categorical variables, paired t-test for continuous variables with normal } \\
\text { distribution, and Wilcoxon Rank sum tests for non-normally distributed continuous variables }\end{array}$} \\
\hline
\end{tabular}

\section{Inpatient Stays}

In the matched cohort, the crude number of all-cause inpatient stays in insulin users was 0.8 (standard deviation [SD] 1.3) per person within 12 months before insulin initiation and 0.7 (SD 1.4) within the 12 months after. Non-insulin users had 0.3 (SD 0.9) and 0.3 (SD 0.8) all-cause inpatient stays per person in the 12 months before and after the matched date, respectively. In the adjusted GEE model (Table 2), insulin users had a higher rate of inpatient stays than non-insulin users (rate ratio [RR]: 2.3 [2.1, 2.4]). However, the rate of inpatient stays did not change after insulin initiation (RR: 1.2 [1.0, 1.5]). No significant difference was found in within-person change in inpatient stays between insulin and noninsulin users (RR: $1.0[0.9,1.1])$. In young adults, insulin users had a greater subsequent increase in inpatient stays after insulin initiation vs. non-insulin users over the same time period (RR: 1.3 [1.1, 1.5]), which was not found in middle-aged adults (RR: $0.9[0.8,1.0]$ ) (Additional file 4). Among insulin users, no association was found between the time to insulin initiation after first-line GLAs and the within-person change of inpatient stays either in the matched cohort (RR: $1.0[1.0,1.0]$, Table 3 ) or in the subgroups of young and middle-aged enrollees. 
Table 3

GEE models for the association between timing of insulin initiation and outcomes

\begin{tabular}{|c|c|c|c|c|}
\hline \multirow[t]{2}{*}{ Parameter } & \multicolumn{2}{|c|}{ Univariate Model } & \multicolumn{2}{|c|}{ Multivariable model } \\
\hline & $\mathrm{RR}(95 \% \mathrm{Cl})$ & $\begin{array}{l}\mathrm{p}- \\
\text { value }\end{array}$ & $\begin{array}{l}\mathrm{RR}(95 \% \\
\mathrm{Cl})\end{array}$ & $\begin{array}{l}\text { p- } \\
\text { value }\end{array}$ \\
\hline \multicolumn{5}{|l|}{ Rate of inpatient stays $¥$} \\
\hline After vs. before insulin initiation* & $\begin{array}{l}0.90(0.81 \\
0.98)\end{array}$ & 0.02 & $\begin{array}{l}2.01(1.43 \\
2.80)\end{array}$ & $\begin{array}{l}< \\
0.01\end{array}$ \\
\hline $\begin{array}{l}\text { Before insulin initiation: } 1 \text { year increase in time to } \\
\text { insulin initiation }\end{array}$ & $\begin{array}{l}0.97(0.94 \\
1.00)\end{array}$ & 0.10 & $\begin{array}{l}1.02(0.99 \\
1.05)\end{array}$ & 0.15 \\
\hline $\begin{array}{l}\text { Interaction term of within person change and time to } \\
\text { insulin initiation } \dagger\end{array}$ & $\begin{array}{l}1.01(0.98 \\
1.04)\end{array}$ & 0.57 & $\begin{array}{l}0.99(0.96 \\
1.03)\end{array}$ & 0.77 \\
\hline \multicolumn{5}{|l|}{ Rate of Emergency room (ER) visits $\S$} \\
\hline After vs. before insulin initiation* & $\begin{array}{l}0.93(0.87 \\
1.00)\end{array}$ & 0.05 & $\begin{array}{l}0.96(0.88 \\
1.05)\end{array}$ & 0.44 \\
\hline $\begin{array}{l}\text { Before insulin initiation: } 1 \text { year increase in time to } \\
\text { insulin initiation }\end{array}$ & $\begin{array}{l}0.98(0.95 \\
1.01)\end{array}$ & 0.22 & $\begin{array}{l}1.00(0.97 \\
1.02)\end{array}$ & 0.76 \\
\hline $\begin{array}{l}\text { Interaction term of within person change and time to } \\
\text { insulin initiation } \dagger\end{array}$ & $1.02(1.00$ & 0.10 & $\begin{array}{l}1.01(0.99 \\
1.04)\end{array}$ & 0.29 \\
\hline
\end{tabular}

RR: Rate ratio

* RRs for those with the average length of time to insulin initiation

† RR for interaction terms were interpreted as the relative rate of within-person changes in healthcare utilization per 1 year increase in time to insulin initiation

‡ Final model for rate of inpatient stays adjusted for race, sex, modified Elixhauser index, hypertension, psychosis, congestive heart failure, nephropathy, neuropathy, micro/macro-vascular complications, use of anticoagulant agents and lipid lowering agents, as well as interaction terms between within-person change and age, Medicaid eligibility categories (disabled or chronic conditions/others), depression, metabolic complications.

$\S$ Final model for rate of ER visits adjusted for age, race, sex, modified Elixhauser index, Medicaid eligibility categories (disabled or chronic conditions/others), hypertension, obesity, depression, psychosis, neuropathy, micro/macro-vascular complications, as well as interaction terms between within-person change and anti-hypertensives, lipid lowering agents.

\section{Emergency Room (er) Visits}

In the matched cohort, the crude numbers of all-cause ER visits within 12 months before and after insulin initiation were 2.2 (standard deviation [SD] 4.0) and 2.2 (SD 4.1) per person for insulin users, respectively. Non-insulin users had 1.4 (SD 3.1) and 1.3 (SD 3.0) all-cause ER visits per person in 12 months before and after the matched date of insulin initiation. In the adjusted GEE model, insulin users had a higher rate 
of ER visit than non-insulin users (RR: $1.5[1.4,1.6])$, Table 2). The number of ER visits decreased within 12 months after the matched date by $5 \%$ (RR: $1.0[0.9,1.0]$ ). No significant difference was found in withinperson change of ER visits between insulin and non-insulin users either in the matched cohort (RR: 1.0 $[1.0,1.1])$ or in the subgroups of young and middle-aged enrollees. Among insulin users, no association was found between the time to insulin initiation and the within-person change of ER visits (RR: 1.0 [1.0, 1.0], Table 3). The results in the subgroup of those with 2 or more refills for insulin vs. non-insulin users were consistent with the primary analysis.

\section{Discussion}

In our claims-based study among young and middle-aged Medicaid enrollees in Pennsylvania, insulin users had more inpatient stays and ER visits than matched non-insulin users after controlling for several baseline demographics, comorbidities, and complications. However, compared with non-insulin users, insulin users had a similar pattern of change in inpatient stays or ER visits within 12 months after insulin initiation vs. before. We found that insulin users in the age group of $18-45$ years had a greater increase in the number of inpatient stays after insulin initiation compared to non-insulin users in the same age group, which has not been addressed in previous studies. Among insulin users, the length of time to insulin initiation after GLAs was not associated with the change in healthcare utilization. To our knowledge, this study is the first to investigate the association between insulin initiation and the subsequent changes in inpatient and ER services in both young and middle-aged Medicaid enrollees who have low-incomes, high levels of disability, and comorbid mental health and physical health conditions [17-19].

We found a higher level of inpatient and ER services utilization in insulin users than non-insulin users regardless of the time from first-line GLAs to insulin initiation, possibly due to several unmeasurable confounders related to diabetes severity. Prior observational studies showed that insulin users had higher glycemic levels and more existing complications, which may yield substantial healthcare utilization and costs [20], than non-insulin users [17-19]. In a study of managed care patients with T2DM on insulin, those who initiated insulin after 1 GLA had more complications than delayed insulin initiation after $\geq 3$ GLAs [9]. These studies may reflect that insulin is initiated reactively to slow down the progression of complications rather than proactively to prevent the occurrence of complications in clinical practice [21]. We employed a matching strategy with propensity scores and covariate adjustments to reduce bias by balancing characteristics before first-line GLAs between insulin and non-insulin users. Nevertheless, our ability to test this hypothesis is limited by the fact that we are unable to adjust for $\mathrm{HbA} 1 \mathrm{c}$ levels, lipid profiles, BMI, duration of diabetes, smoking status and other disease markers related to diabetes severity at baseline. One study reported insulin users in health maintenance organizations (HMO) had more ER visits than those using sulfonylureas cross-sectionally; however, the difference attenuated to the null after adjusting for $\mathrm{HbA} 1 \mathrm{c}$ levels, diabetes duration, and baseline diabetes severity measured by self-reported symptoms, disease manifestations, and diagnosed complications [22]. This finding further supports that the higher level of inpatient and ER services utilization in insulin users in our study may be the result of insulin users having a higher level of comorbidity, potentially unmeasured in administrative data. 
Our finding regarding a lack of association between insulin initiation and within-person reductions in ER visits is consistent with prior work. One study in individuals with T2DM from an HMO showed the number of ER visits did not change within 12 months after insulin initiation in insulin users adjusting for withinperson changes in non-insulin users (adjusted pre-post ER visits change per year: 0.1 [0.0-0.2]) [22]. However, this study was conducted in a commercial HMO, with fewer racial/ethnic minorities and higher income distribution compared to Medicaid enrollees [22]. Medicaid enrollees were more likely to use ER services compared with privately insured populations, which is partially explained by their poor access to primary, specialty and other outpatient health care [23]. Some projects have been implemented in Medicaid since 2012, which included providing supportive housing for homeless adults and implementing case management in frequent ER users, to re-direct non-urgent ER cares to the most appropriate settings [24]. An HMO study found that insulin initiation was related to an increase in outpatient visits probably due to additional glucose monitoring, insulin dose adjustments or foot and eye examinations (adjusted pre-post outpatient visits change per year: 3.1[1.9-4.0]) [22]. Whether those projects to control non-urgent use of ER or an increased use of outpatient visits after insulin initiation was related to the reduction in ER visits in our study is unclear and represents a direction of further study.

Our study showed insulin users aged $\leq 45$ years had an increase in inpatient stays during 12 months after insulin initiation, which has not been addressed in previous studies among adults with T2DM. Previous studies showed that after insulin initiation, the proportion of those with inpatient visits was reduced by $10 \%$ in privately insured individuals with a mean age of 55 [9], and the average number of inpatient visits in 9 months decreased by 0.16 among middle-aged adults [10]. However, neither of these studies included non-insulin users as comparators and examined the association between insulin use and inpatient services in adults aged $\leq 45$ years. Young adults with T2DM may be characterized by an accelerated loss of $\beta$-cell function, a higher prevalence of obesity, and a higher risk of microalbuminuria compared to middle-aged adults with T2DM [25-28]. The evidence has previously indicated a more aggressive disease progression in young adults vs. middle-aged adults, which may yield more inpatient stays. In young adults with T2DM, insulin is usually initiated for those with marked hyperglycemia (A1C $\geq 8.5 \%$ ) and any symptoms of diabetes (e.g. polyuria, polydipsia, nocturia, and/or weight loss) [29]. While we were limited to diagnoses captured in claims data, it may be that young insulin users in our cohort had more severe diabetes disease progression than non-insulin users.

Our study is the first to observe the effect of young vs. middle age on the association between insulin initiation and healthcare utilization in low-income Medicaid enrollees. We were able to address the withinperson change of healthcare utilization adjusting for underlying changes in non-insulin users, which is rarely examined in previous studies. Additionally, our Medicaid database obtained directly from the Commonwealth captures drug and healthcare utilization from beneficiaries in managed care plans, which is not always available in Medicaid databases from the Centers for Medicare and Medicaid Services (CMS). The comprehensive reporting system for encounter data in the Pennsylvania Department of Human Service (PADHS) provides a reliable and valid measure of utilization from managed care. 
A key limitation of this study is that some potential confounders were not available in our claims data, especially clinical laboratory data (e.g. HbA1c, lipid profiles, and weight), health-related behavior (e.g. smoking), and physicians' referral behavior. In addition, healthcare utilization was evaluated for 1 year before and after insulin initiation, so an extended time frame may be needed to observe long-term change of healthcare utilization after insulin initiation. Moreover, using the real-world data, we were not able to determine if insulin initiation slowed down the increasing rate of healthcare utilization in the insulin users compared to those in whom insulin was not initiated. Finally, our study population is representative of a typical Medicaid population in Pennsylvania. Given variation in Medicaid policy across the states our results may not generalize to other states.

\section{Conclusions}

In T2DM Medicaid enrollees, neither insulin initiation nor the time to insulin initiation from first line therapy was found to be related to within-person change in inpatient stays or ER visits accounting for several baseline characteristics. However, in T2DM Medicaid enrollees with insulin therapy aged $\leq 45$ years, inpatient use increased after insulin initiation at a higher rate than non-insulin users, possibly due to a more aggressive disease progression. Further studies of investigating the contributors to the increased inpatient use after insulin initiation are needed targeting young insulin users with T2DM in Medicaid.

\section{Declarations}

\section{Ethics approval and consent to participate}

The University of Pittsburgh IRB reviewed this study (IRB\#: PR012100227) and ruled that since it did not involve any interaction with human subjects, only review of existing data, it did not require formal ethics approval and could be designated as "exempt". Per the University of Pittsburgh IRB the study met all the necessary criteria for an exemption under section:

45 CFR 46.101(b) (4) existing data, documents, or records

Additionally, the Pennsylvania Department of Human Services (PADHS) granted formal permission to access their databases through separate data use agreements with the University of Pittsburgh.

Consent for publication: Not applicable.

Availability of data and materials: The data that support the findings of this study are available from PADHS but restrictions apply to the availability of these data, which were used under license for the current study, and so are not publicly available.

Competing interests: The authors declare that they have no competing interests. 
Funding: This study and medical writing for this manuscript were funded by the National Heart Lung and Blood institute (NHLBI) through a research grant (R01 HL119246). NHLBI had no role in the design of the study, the writing of the manuscript, or in the collection, analysis, or interpretation of the data.

Author Contributions: LX wrote the manuscript and analyzed study data. LX, ES, RB, JZ, TC and JD contributed to the study concept and design. LX, ES, RB and JD interpreted study data. ES, RB, JZ, TC, DK and JD provided critical review and edits to the manuscript and gave final approval before submission. J.D. is the guarantor of this work and, as such, had full access to all the data in the study and takes responsibility for the integrity of the data.

Acknowledgments: The authors thank Jie Li and Aiju Men for expert programming.

\section{References}

1. Dall TM, Yang W, Halder P, Franz J, Byrne E, Semilla AP, Chakrabarti R, Stuart B: Type 2 diabetes detection and management among insured adults. Population health metrics 2016, 14:43.

2. American Diabetes Association: 8. Pharmacologic Approaches to Glycemic Treatment.Diabetes care 2017, 40:S64-S74.

3. Shrestha SS, Honeycutt AA, Yang W, Zhang P, Khavjou OA, Poehler DC, Neuwahl SJ, Hoerger TJ: Economic Costs Attributable to Diabetes in Each U.S. State. Diabetes Care 2018, 41:2526-2534.

4. Li R, Bilik D, Brown MB, Zhang P, Ettner SL, Ackermann RT, Crosson JC, Herman WH: Medical costs associated with type 2 diabetes complications and comorbidities. The American journal of managed care 2013, 19:421-430.

5. Wagner EH, Sandhu N, Newton KM, McCulloch DK, Ramsey SD, Grothaus LC: Effect of improved glycemic control on health care costs and utilization.JAMA 2001, 285:182-189.

6. Weng J, Li Y, Xu W, Shi L, Zhang Q, Zhu D, Hu Y, Zhou Z, Yan X, Tian H et al: Effect of intensive insulin therapy on beta-cell function and glycaemic control in patients with newly diagnosed type 2 diabetes: a multicentre randomised parallel-group trial. Lancet 2008, 371(9626):1753-1760.

7. Holman RR, Paul SK, Bethel MA, Matthews DR, Neil HAW: 10-year follow-up of intensive glucose control in type 2 diabetes.New England journal of medicine 2008, 359:1577-1589.

8. Luo J, Avorn J, Kesselheim AS: Trends in medicaid reimbursements for insulin from 1991 through 2014. JAMA Internal Medicine 2015, 175:1681-1686.

9. Levin PA, Zhou S, Gill J, Wei W: Health Outcomes Associated with Initiation of Basal Insulin After 1, 2, or $\geq 3$ Oral Antidiabetes Drug(s) Among Managed Care Patients with Type 2 Diabetes. Journal of Managed Care \& Specialty Pharmacy 2015, 21:1172-1182.

10. Rosenblum MS, Kane MP: Analysis of cost and utilization of health care services before and after initiation of insulin therapy in patients with type 2 diabetes mellitus. Journal of managed care pharmacy : JMCP 2003, 9:309-316. 
11. Grubesic TH, Matisziw TC: On the use of ZIP codes and ZIP code tabulation areas (ZCTAs) for the spatial analysis of epidemiological data. International Journal of Health Geographics 2006, 5:58.

12. Glasheen WP, Renda A, Dong Y: Diabetes Complications Severity Index (DCSI)-Update and ICD-10 translation. Journal of Diabetes and its Complications 2017, 31:1007-1013.

13. Elixhauser A, Steiner C, Harris DR, Coffey RM: Comorbidity Measures for Use with Administrative Data. Medical Care 1998, 36:8-27.

14. Data Matching - Optimal and Greedy. In.: NCSS Statistical Software.

15. Other Inpatient Stays [https://www.resdac.org/cms-data/variables/other-inpatient-stays]

16. Hennessy S, Leonard CE, Freeman CP, Deo R, Newcomb C, Kimmel SE, Strom BL, Bilker WB: Validation of diagnostic codes for outpatient-originating sudden cardiac death and ventricular arrhythmia in Medicaid and Medicare claims data. Pharmacoepidemiol Drug Saf 2009, 19:555-562.

17. Kostev K, Rathmann W: Influence of macro- and microvascular comorbidity on time to insulin initiation in type 2 diabetes patients: a retrospective database analysis in Germany, France, and UK.Primary care diabetes 2013, 7:167-171.

18. Mast R, Jansen APD, Walraven I, Rauh SP, Van Der Heijden AAWA, Heine RJ, Elders PJM, Dekker JM, Nijpels G, Hugtenburg JG: Time to insulin initiation and long-term effects of initiating insulin in people with type 2 diabetes mellitus: The Hoorn Diabetes Care System Cohort Study. European Journal of Endocrinology 2016, 174:563-571.

19. Roumie CL, Greevy RA, Grijalva CG, Hung AM, Liu X, Murff HJ, Elasy TA, Griffin MR: Association between intensification of metformin treatment with insulin vs sulfonylureas and cardiovascular events and all-cause mortality among patients with diabetes.JAMA 2014, 311:2288-2296.

20. Alva ML, Gray A, Mihaylova B, Leal J, Holman RR: The impact of diabetes-related complications on healthcare costs: new results from the UKPDS (UKPDS 84).Diabetic medicine : a journal of the British Diabetic Association 2015, 32:459-466.

21. Brown JB, Nichols GA: Slow response to loss of glycemic control in type 2 diabetes mellitus.American journal of managed care 2003, 9:213-217.

22. Hayward RA, Manning WG, Kaplan SH, Wagner EH, Greenfield S: Starting insulin therapy in patients with type 2 diabetes: effectiveness, complications, and resource utilization.JAMA 1997, 278:16631669.

23. Medicaid and CHIP Payment and Access Commission: Revisiting Emergency Department Use in Medicaid. In. Washington, DC: MACPAC; 2014.

24. Mann C: Reducing Nonurgent Use of Emergency Departments and Improving Appropriate Care in Appropriate Settings. In.; 2014.

25. Al-Saeed AH, Constantino MI, Molyneaux L, D'Souza M, Limacher-Gisler F, Luo C, Wu T, Twigg SM, Yue DK, Wong J: An inverse relationship between age of type 2 diabetes onset and complication risk and mortality: The impact of youth-onset type 2 diabetes. Diabetes Care 2016, 39:823-829. 
26. Hillier TA, Pedula KL: Complications in young adults with early-onset type 2 diabetes: losing the relative protection of youth.Diabetes Care 2003, 26:2999-3005.

27. Lascar N, Brown J, Pattison H, Barnett AH, Bailey CJ, Bellary S: Type 2 diabetes in adolescents and young adults. The Lancet Diabetes \& Endocrinology 2018, 6:69-80.

28. Song SH, Hardisty CA: Early onset type 2 diabetes mellitus: A harbinger for complications in later years-clinical observation from a secondary care cohort. QJM : monthly journal of the Association of Physicians 2009, 102(11):799-806.

29. Arslanian S, Bacha F, Grey M, Marcus MD, White NH, Zeitler P: Evaluation and Management of YouthOnset Type 2 Diabetes: A Position Statement by the American Diabetes Association. Diabetes Care 2018, 41(12):2648-2668.

\section{Figures}

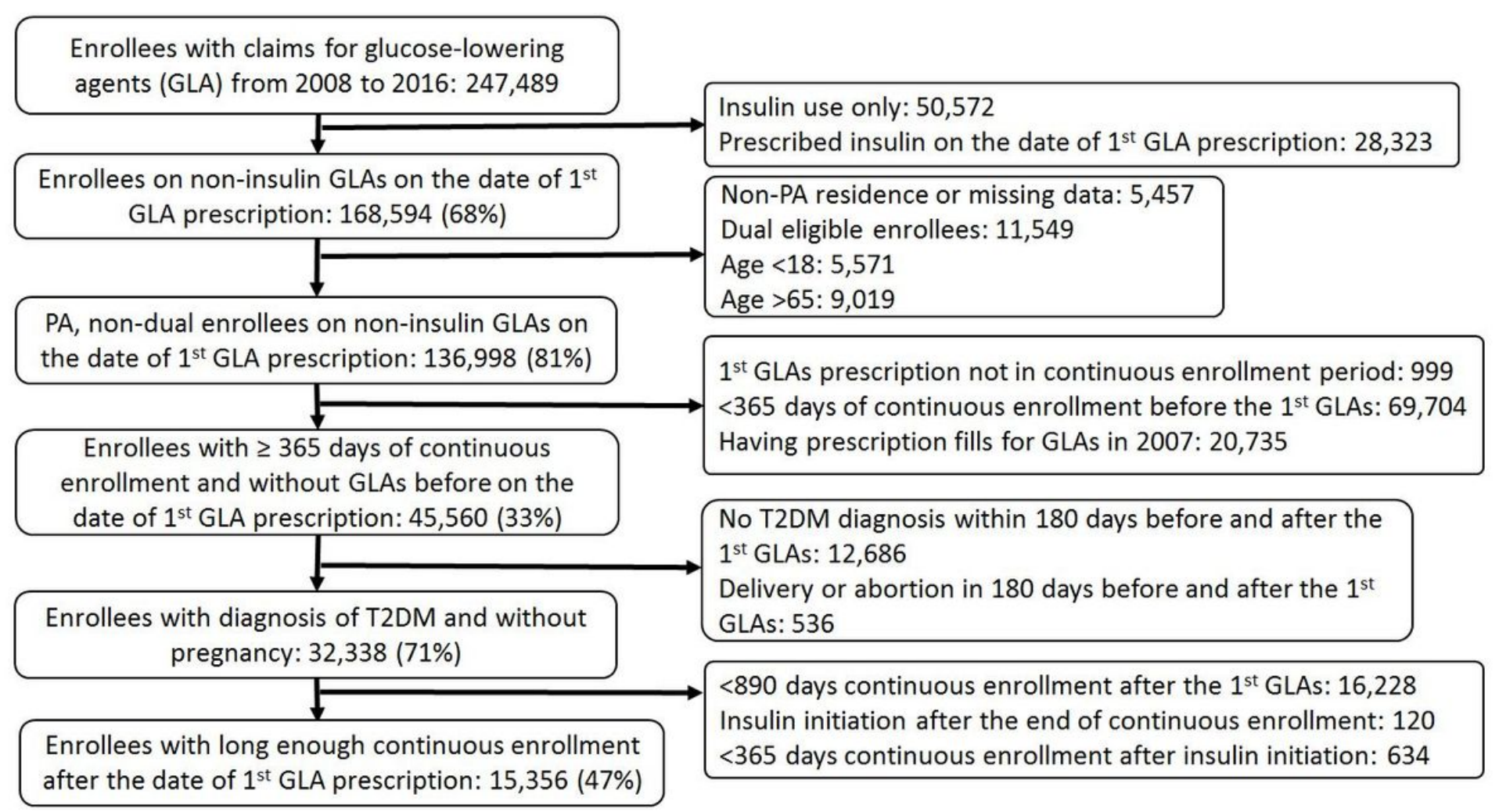

\section{Figure 1}

Flow chart of the eligible patients with type 2 diabetes (T2DM) GLA: glucose-lowering agents. 


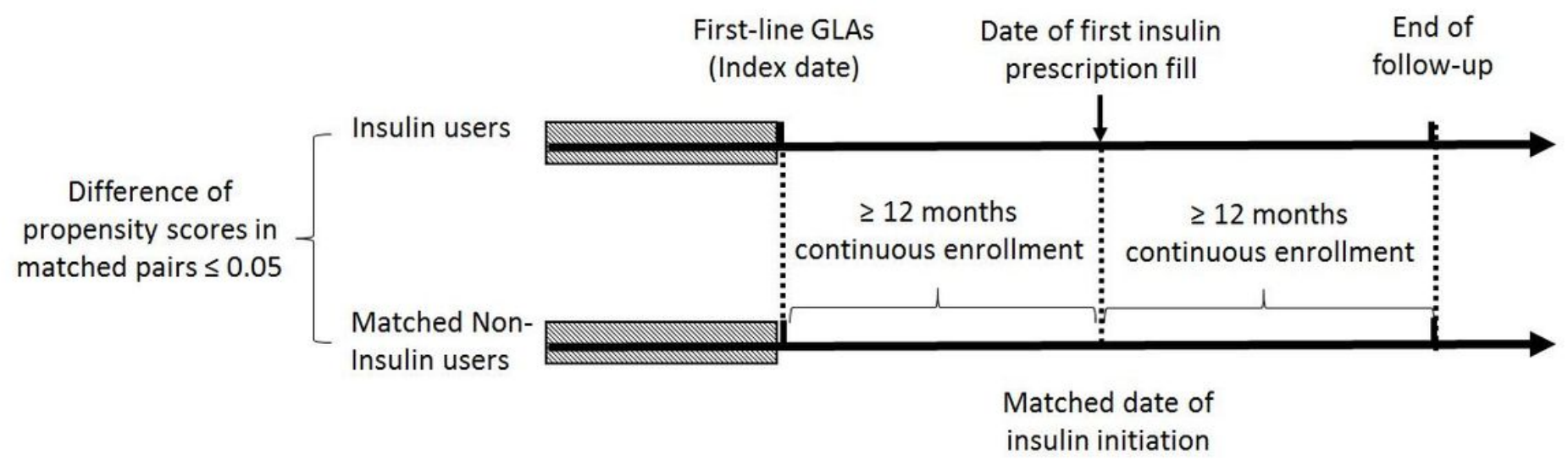

Figure 2

Matching process A hierarchical 1:3 matching strategy: non-insulin users were matched with insulin users on a) the index date (the date of first line GLAs) \pm 5 days, b) continuous enrollment within 12 months before and after the matched date of insulin initiation, and c) propensity scores (PS) with a deviation of 0.05 on the PS scale. For non-insulin users, the available period for matching was from the first-line GLAs through the date 12 months before the end of follow up. The measurement period for baseline covariates was 12 months before first-line GLA (bar with strips).

\section{Supplementary Files}

This is a list of supplementary files associated with this preprint. Click to download.

- Additionalfile1.docx

- Additionalfile4.docx

- Additionalfile3.docx

- Additionalfile2.docx 